XVI CONGRESO LATINOAMERICANO DE PATOLOGÍA DE LA CONSTRUCCÍN
XVIII DE CONTROL DE CALIDAD EN LA CONSTRUCCIÓN

\title{
Diagnóstico y patología del puente de hierro sobre el río Jatibonico.
}

\author{
R. Pérez *1, J. Nápoles ${ }^{2}$ \\ *Raimundo F. Pérez Delgado: mundi@eniavc.cu \\ ${ }^{1}$ Departamento de Geotecnia, Empresa Nacional de Investigaciones Aplicadas UIC Villa Clara, Santa Clara, Cuba \\ ${ }^{2}$ Departamento de Geotecnia, Empresa Nacional de Investigaciones Aplicadas UIC Villa Clara, Santa Clara, Cuba
}

\begin{abstract}
RESUMEN
Las visibles muestras de deterioro del puente de hierro del $\mathrm{km} 416$ de la Carretera Central, con vibraciones acentuadas entre otras patologías, requirieron de estudios con el objetivo de diagnosticar su estado actual con evaluación del desempeño. El trabajo enfocado a la superestructura con análisis de estribos, elementos de apoyo, cerchas, tablero y arriostres, consigue el levantamiento estructural y caracterización de sus materiales con incertidumbre propia del laboreo sin cierre de la vía, sin acceso a determinadas uniones. Pionero entre otros trabajos similares requeridos en esta vía con casi 100 años de explotación y deficiente mantenimiento puede constituir una guía de trabajo. La infraestructura vial indicador de salud económica, justifica avizorar anomalías implementando las medidas de restauración a tiempo.
\end{abstract}

Palabras clave: diagnóstico; puente de hierro; elementos estructurales 


\section{RESUME}

The visible signs of deterioration of the iron bridge of $\mathrm{km} 416$ of the Central Highway, with accentuated vibrations among other pathologies, required studies in order to diagnose its current state with performance evaluation. The work focused on the superstructure with analysis of abutments, support elements, trusses, deck and bracing, achieves the structural lifting and characterization of its materials with the uncertainty of working without closure of the road, without access to certain joints. Pioneer among other similar jobs required on this road with almost 100 years of operation and poor maintenance can constitute a work guide. The road infrastructure, an economic health indicator, justifies foreseeing anomalies by implementing restoration measures on time.

Keywords: diagnosis; iron bridge; structural elements

\section{RETOMAR}

Os visíveis sinais de deterioração da ponte de ferro do km 416 da Rodovia Central, com vibrações acentuadas entre outras patologias, exigiram estudos para diagnosticar seu estado atual com avaliação de desempenho. O trabalho centrado na superestrutura com análise de pilares, elementos de apoio, treliças, tabuleiro e travessas, consegue o levantamento estrutural e caracterização dos seus materiais com a incerteza típica de trabalhar sem fecho de estrada, sem acesso a determinadas juntas. Pioneirismo entre outros trabalhos semelhantes exigidos nesta estrada com quase 100 anos de operação e má manutenção pode constituir um guia de trabalho. A infraestrutura rodoviária, um indicador de saúde econômica, justifica a previsão de anomalias com a implementação pontual de medidas de restauração.

Palavras-chave: diagnóstico; ponte de ferro; elementos estruturais 


\section{INTRODUCCIÓN}

La tarea requería un levantamiento detallado de la superestructura, dimensiones y sección transversal de los elementos componentes, posibles deformaciones de estos con magnitud y causas, calidad del acero, resistencia, límite de elasticidad y grado de corrosión, incluyendo los aparatos de apoyo, así como funcionalidad de estos últimos, se involucraron servicios topogeodésicos, de patología y diagnóstico, ensayos de campo y laboratorio y los trabajos de gabinete. La obra estudiada, un puente de estructuras metálicas, de $76.20 \mathrm{~m}$ de longitud en una sola luz, salvando alrededor de $12 \mathrm{~m}$ de desnivel en cause pronunciado con abundante vegetación, sin acceso vehicular a este y peatonal sólo a los dos primeros tramos de su ribera norte y uno de su ribera sur desde los estribos, no disponía de planos estructurales ocasionando incrementos considerables en los trabajos decampo y de oficina. El difícil acceso y la carencia de andamios y equipamiento que permitiera acceder a toda la estructura, unidos a la imposibilidad de cerrar la vía al tráfico durante los trabajos de campo, si bien no afectaron el levantamiento estructural si introducen en el levantamiento de lesiones subjetividad, imprecisiones e incertidumbre que le dan carácter de preliminar al estudio con aspectos pendientes de verificación en etapa ejecutiva, además del indiscutible conservador criterio técnico.

\section{DESCRIPCION GENERAL DEL PUENTE}

El objeto de estudio es un puente de estructura metálica, con alrededor de 90 años de servicio. Su estructura, simétrica en ambas direcciones, la forman dos cerchas longitudinales de $76.505 \mathrm{~m}$ de largo por $12.80 \mathrm{~m}$ de alto, compuestas por un cordón inferior horizontal y un cordón superior en forma de arco unidos mediante montantes y diagonales, 11 vigas principales transversales espaciadas a $7.62 \mathrm{~m}$ dando lugar a 10 tramos con iguales dimensiones, 60 vigas longitudinales secundarias a razón de 6 por tramos, espaciadas a $1.25 \mathrm{~m}$ y por último 10 crucetas inferiores, 8 crucetas superiores, 2 portales extremos y 7 contravientos interiores con elementos diagonales, todos a manera de cierre y arriostre; estos cierres de la estructura garantizan un gálibo de $5.00 \mathrm{~m}$. Esta estructura descansa en cuatro elementos de apoyo que permiten el giro todos y dos de ellos, en el estribo sur, el desplazamiento longitudinal además de trasmitir las cargas a la infraestructura limitada a dos estribos en forma de muros de contención, de gravedad, de hormigón masivo y con aletones inclinados. La calzada presenta pavimento rígido de hormigón armado fundido "in situ", cubierto con adoquines y capa de rodadura de hormigón asfáltico para el tráfico vehicular, y aceras laterales también de hormigón armado, con barandas exteriores, para el tráfico peatonal. (Ver Figura 1). Con el propósito de facilitar los trabajos y hacer asequible la comprensión de los mismos se establece la siguiente nomenclatura para designar ejes, elementos y uniones entre otros componentes según esquema siguiente: 


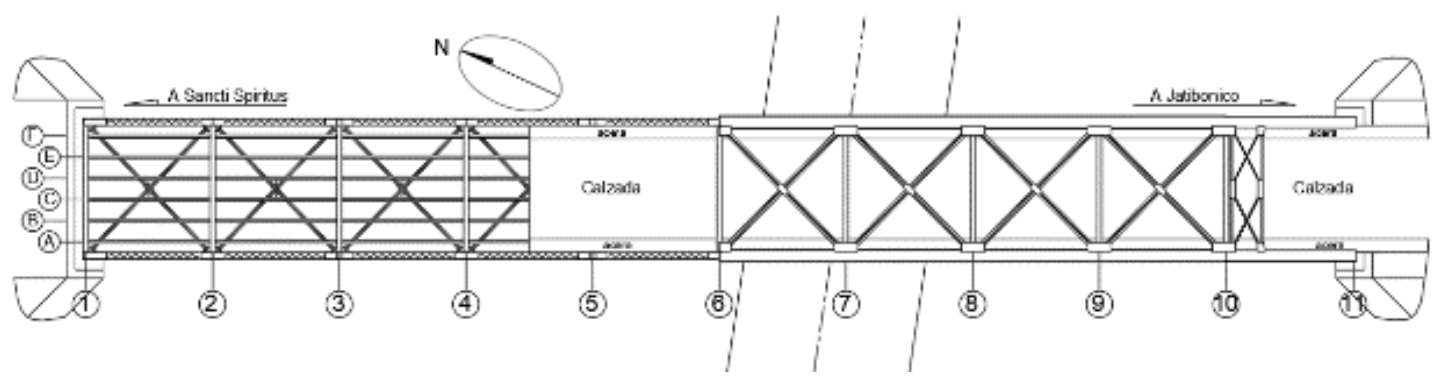

Figura 1: Esquema con la nomenclatura de los elementos

De esta forma las cerchas se definirán como A' y F' a tono con el eje longitudinal próximo y sus componentes como son cordón superior e inferior, montantes y diagonales serán referidos a los ejes transversales correspondientes, así también se denominarán apoyos, uniones y elementos de cierre.

\section{LEVANTAMIENTO ESTRUCTURAL}

Para el levantamiento estructural de toda la estructura se midieron con cintas métricas, disto y pie de rey las secciones de cada uno de los elementos componentes incluyendo la disposición y espaciamiento de los mismos, así como de las cabezas y orificios de los remaches, lo que unido al resultado del escaneo posibilitaron la confección de los planos de la estructura. (Ver Figura 2)
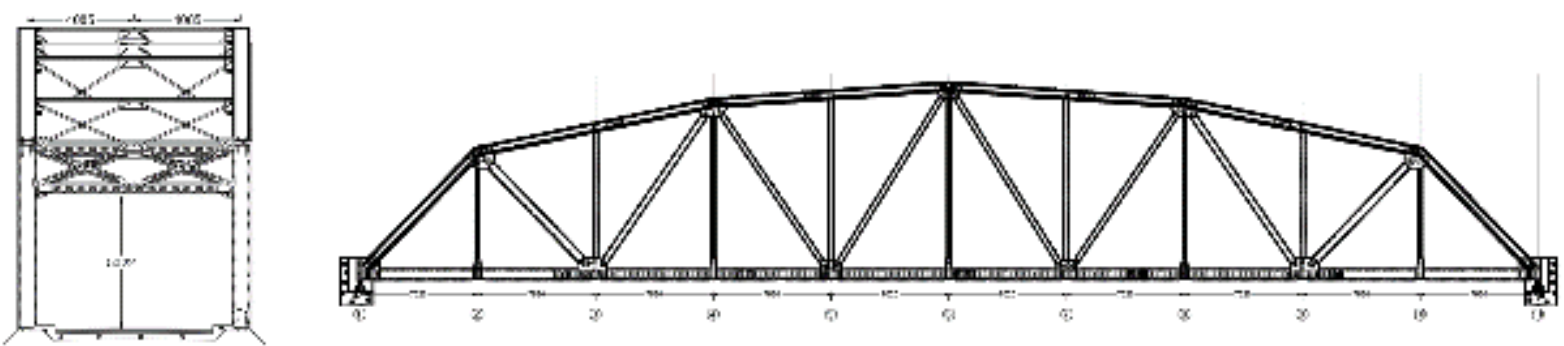

Figura 2: Vistas lateral y frontal del puente

\section{ENSAYOS "IN SITU”}

\subsection{Esclerometría+}

Se evaluaron un total de 18 puntos de esclerometría en losa a razón de 10 disparos por punto, ubicados según muestra el esquema que sigue; limitados en cantidad por tener acceso a solo tres tramos (1 2, 2 3 y 10 11). Ver Figura 3. 

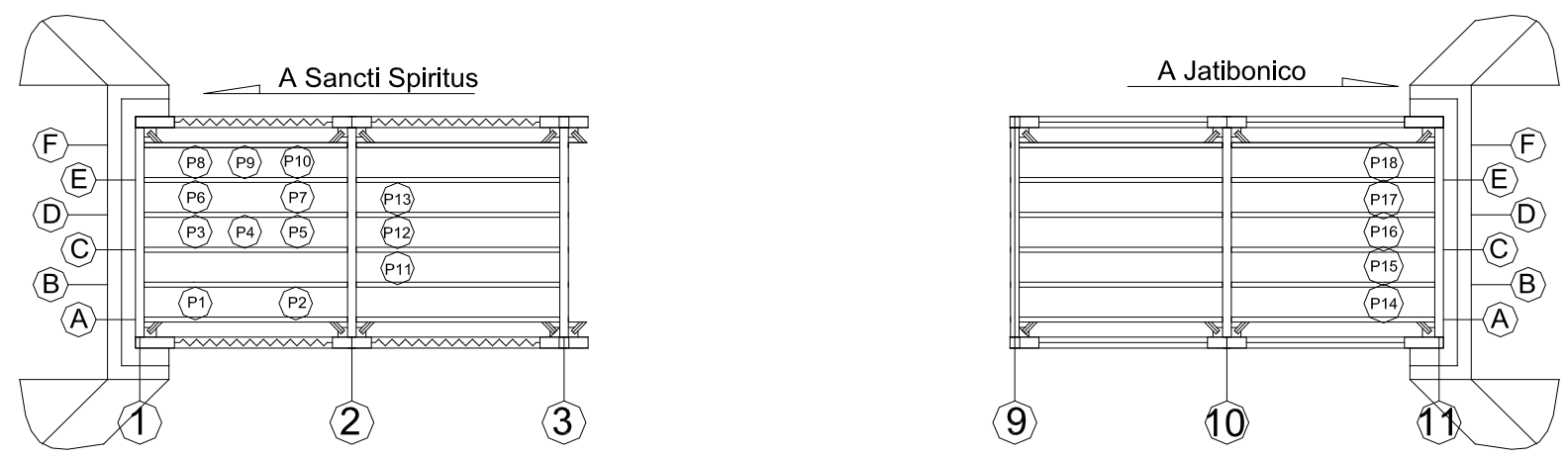

Figura 3: Posiciones de los ensayos de esclerometría

Como resultado se tiene el promedio de la resistencia característica superficial del hormigón de la losa del Puente de Jatibonico fue de 36.1 MPa, resultando valores superiores a $20 \mathrm{MPa}$ para un hormigón de más de 28 días, por lo tanto, cumple con lo establecido en la NC 250:2005, Requisitos de Durabilidad para el Diseño y Construcción de Edificaciones y Obras Civiles de Hormigón Estructural y en la NC 120:2014. Hormigón Hidráulico. Especificaciones, para un nivel de agresividad bajo.

\subsection{Pacometría}

Aun cuando en varias zonas los efectos de la corrosión del refuerzo de la losa provocaron pérdidas de recubrimiento con exposición del mismo, se realizaron mediciones del espaciamiento y diámetro en zonas sin pérdida de recubrimiento verificando la uniformidad de su disposición en los diferentes paños cuyos resultados indican mallas con aceros de temperatura ø $12.7 \mathrm{~mm}(1 / 2$ ") espaciados a $120 \mathrm{~mm}$, con incremento del mismo hacia el centro del paño a $220 \mathrm{~mm} \mathrm{y}$ recubrimiento de $36 \mathrm{~mm}$; el acero de carga también de ø $12.7 \mathrm{~mm}(1 / 2$ ") está espaciado a $80 \mathrm{~mm}$ con aumento hacia el centro del paño a $120 \mathrm{~mm}$ y recubrimiento de $25 \mathrm{~mm}$.

\subsection{Ensayos de resistencia a tracción y doblado}

Se realizaron 8 ensayos de tracción y doblado a muestras de tres elementos metálicos diferentes previamente preparadas cuyos resultados se muestran en la Tabla 1:

Tabla 1: Resultados de ensayos de resistencia a tracción y doblado

\begin{tabular}{|c|c|c|c|c|c|c|}
\hline $\begin{array}{l}\text { Tipo de } \\
\text { elemento }\end{array}$ & $\begin{array}{c}\mathrm{N}^{0} \text { de } \\
\text { muestra }\end{array}$ & $\begin{array}{c}\text { Masa } \\
(\mathrm{g})\end{array}$ & $\begin{array}{c}\text { L- } \\
\text { Largo } \\
(\mathbf{m m})\end{array}$ & $\begin{array}{l}\text { Re-Tensión de } \\
\text { fluencia (MPa) }\end{array}$ & $\begin{array}{c}\text { Rm-Resistencia } \\
\text { a tracción } \\
(\mathrm{MPa})\end{array}$ & $\begin{array}{c}\text { d-Elongación después } \\
\text { de la rotura en } 200 \mathrm{~mm} \\
(\%)\end{array}$ \\
\hline \multirow{3}{*}{$\begin{array}{c}\text { Vigas } \\
\text { principales }\end{array}$} & 1 & 1767 & \multirow{3}{*}{450} & 260 & 404 & 2 \\
\hline & 2 & 1750 & & 381 & 388 & 2 \\
\hline & 3 & 1787 & & 260 & 390 & 2 \\
\hline \multirow{2}{*}{$\begin{array}{c}\text { Vigas } \\
\text { secundarias }\end{array}$} & 1 & 1930 & \multirow{2}{*}{480} & 292 & 450 & 3 \\
\hline & 2 & 1791 & & 271 & 427 & 3 \\
\hline \multirow{3}{*}{$\begin{array}{l}\text { Crucetas } \\
\text { inferiores }\end{array}$} & 1 & 1450 & \multirow{3}{*}{380} & 316 & 474 & 3 \\
\hline & 2 & 1439 & & 342 & 466 & 3 \\
\hline & 3 & 1436 & & 329 & 450 & 3 \\
\hline
\end{tabular}

Por limitaciones del ensayo no se determinan módulos de elasticidad. 


\section{LEVANTAMIENTO PATOLÓGICO}

El levantamiento de lesiones de manera organoléptica permitió la localización de daños en lugares asequibles o a distancia, que fueron volcados a los croquis o dibujos previamente confeccionados, asignan-do un nivel de deterioro a partir de una escala preestablecida como sigue:

1) corrosión sin pérdida de sección.

2) corrosión con pérdida de sección, pero cumple su función.

3) corrosión con pérdida de sección y no cumple su función.

4) pérdida del remache.

5) deformaciones excesivas.

\subsection{Elementos de infraestructura}

La infraestructura está formada sólo por estribos, rectangulares, cerrados, de hormigón masivo, con funciones de muro de contención de gravedad, con aletones, de aproximadamente 10.90x2.20 $\mathrm{m}$ y alturas de 5 7 $\mathrm{m}$ desde el terreno natural sin incluir los aletones que, con inflexión de $30^{\circ}$ se extienden en los laterales con pendiente 3:2. Estos elementos presentan un escalón interior de área 9.90x1.70 m para el apoyo de la estructura. (Ver fotos estribos nno y sse).

Desde el punto de vista geotécnico no presenta manifestaciones de asentamiento ni giros o desplome en los mismos, indicando que sus bases o cimientos alcanzan el estrato resistente e indeformable, sin amenazas de socavación; tampoco muestran signos de deterioro como grietas, fisuras, pérdidas de sección u otros asociados a su explotación y el paso del tiempo, excepto en la parte superior del muro hacia Jatibonico, que muestra, en la parte este e interior una grieta local inclinada al parecer provocada por una junta deficiente entre la losa de pavimento y el muro que impida la trasmisión de los efectos de las vibraciones de la estructura al muro; y en la parte oeste interior del propio estribo que exhibe otra grieta inclinada de mayor extensión y a menos de 40 $\mathrm{cm}$ del borde vinculada a estas mismas acciones además del pase de instalaciones. (Ver foto Grieta de borde de estribo sse).

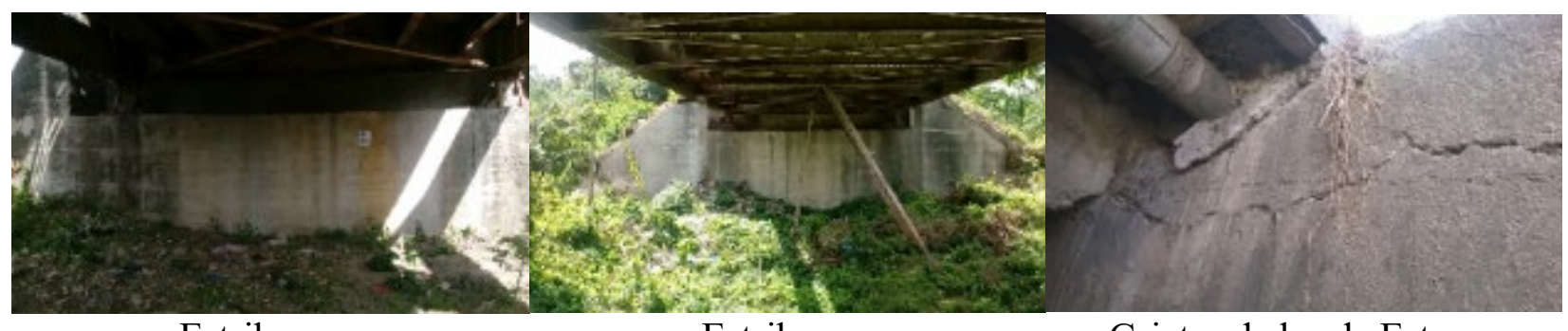

Estribo nno

Estribo sse

Grietas de borde Est. sse

\subsection{Elementos de apoyo}

Los elementos de apoyo, encargados de trasmitir las cargas de la estructura a la infraestructura apoyando en un escalón de $2.20 \mathrm{~m}$ en los estribos para soportarlos y lograr la nivelación entre la estructura y el resto de la vía, constituyen además articulaciones que permiten el giro, y giro con desplazamiento los ubicados en la ribera de Jatibonico. Mediante una articulación cilíndrica de $46.5 \times 7.5 \mathrm{~cm}$ que une dos elementos piramidales metálicos y robustos fijados a través de cuatro pernos de $3.6 \mathrm{~cm}$ de diámetro en cada caso a la estructura metálica y a la infraestructura de hormigón se logra el giro, mientras que los apoyos $11 \mathrm{~A}$ y $11 \mathrm{~F}$ del extremo del lado de Jatibonico, además permiten desplazamiento a través del elemento piramidal inferior que forma otra 
articulación que facilita el deslizamiento horizontal con relación a un tercer elemento que está fijado al estribo también con cuatro pernos de $3.6 \mathrm{~cm}$. (Ver fotos 1A, 1F, 11A y 11F y Figura 4).

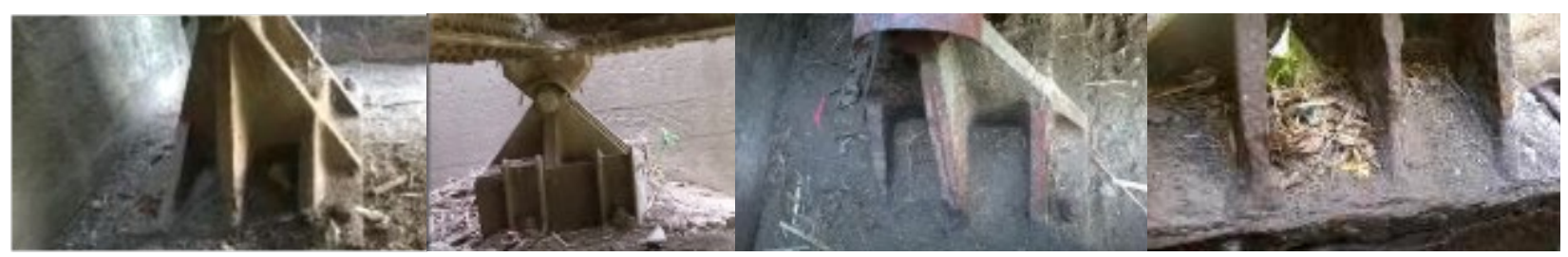

Apoyos $1 \mathrm{~A}$ y $1 \mathrm{~F} \quad$ Apoyos $11 \mathrm{~A}$ y $11 \mathrm{~F}$

Pérdidas de sección
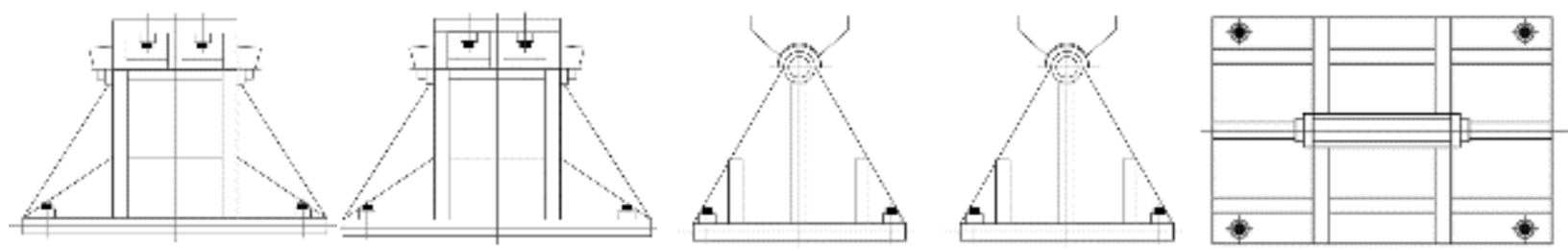

Figura 4: Diferentes vistas de los elementos de apoyo

\subsection{Elementos de superestructura}

La superestructura, constituida por elementos conformados con perfiles y láminas o planchas en casi la totalidad de los casos, excepto las vigas secundarias formadas por un perfil I, presenta secciones con dimensiones detalladas a continuación que en ocasiones son reforzadas convirtiéndose en otra sección como son los casos del cordón inferior de la cercha y las vigas principales que incrementan su inercia y rigidez para sus tramos más solicitados. En la práctica los componentes fundamentales de la estructura son: en las cerchas (cordón inferior, cordón superior, montantes y diagonales), el tablero (vigas principales, vigas secundarias y losa de hormigón armado) y elementos de cierre (portales, crucetas y contra-vientos). (Ver Figuras 5, 6 y 7).

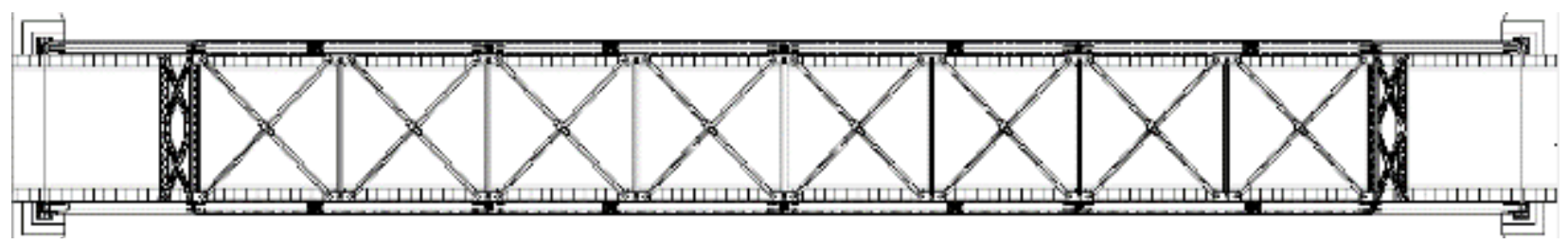

Figura 5: Vista superior

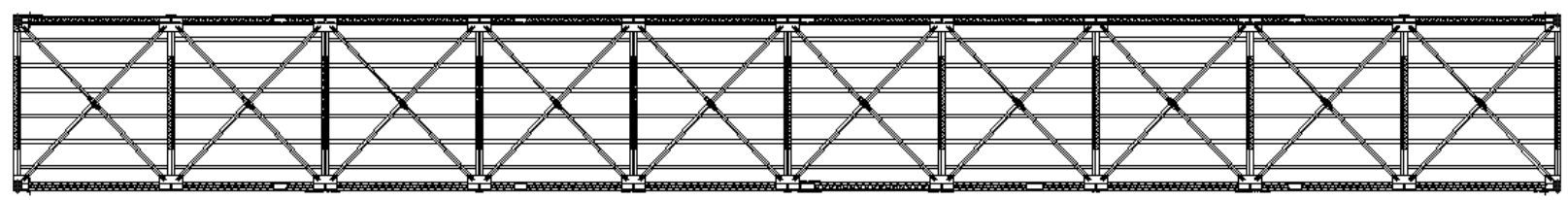

Figura 6: Vista inferior

En todos los casos estos elementos, de conformar secciones cerradas, secciones cajón, presentan una o dos de sus caras relativamente abiertas con riostras, travesaños y elementos de cruceta fijados con remaches, facilitando su revisión y mantenimiento, aunque próximo a las uniones incrementa su rigidez con tramos de láminas que lo dificultan. 
De modo general se han definido 10 perfiles base además de los elementos laminares de dimensiones y espesores variables 6.35, 8, 10, 12, 14, 16, 18 y 20 mm; la conformación de estos elementos se logra con costuras de remaches mientras que las uniones entre estos se consiguen con láminas y cartabones unidos también con remaches de varias dimensiones sin definición exacta en este estudio.

A manera de ejemplo se muestra una representación de uno de los elementos de cada condición en cuanto a levantamiento estructural y de lesiones, destacando que tanto en los elementos conformados como en las uniones la distribución de los remaches y su estado de conservación resultan decisivos en la evaluación de su comportamiento.

- Las uniones entre elemento de apoyo, cordones superior e inferior, viga principal y cruceta inferior se logran mediante dos planchas verticales de $2 \mathrm{~m}^{2}$ y $20 \mathrm{~mm}$ de espesor unidas entre sí con un perfil "I" conformado en su interior y otra lámina de fondo de 750x750x10 mm. A las dos planchas verticales llega el cordón inferior introduciéndose para ensamblar, mientras que el cordón superior lo hace envolviendo estos elementos de unión. En el caso de la viga principal empalma con el elemento de unión interior mediante angulares y remaches y a la lámina horizontal mediante remaches a la que se le une también la cruceta inferior correspondiente. La parte superior del apoyo se une mediante 4 pernos de $36 \mathrm{~mm}$ de diámetro. (Ver Figura 7)
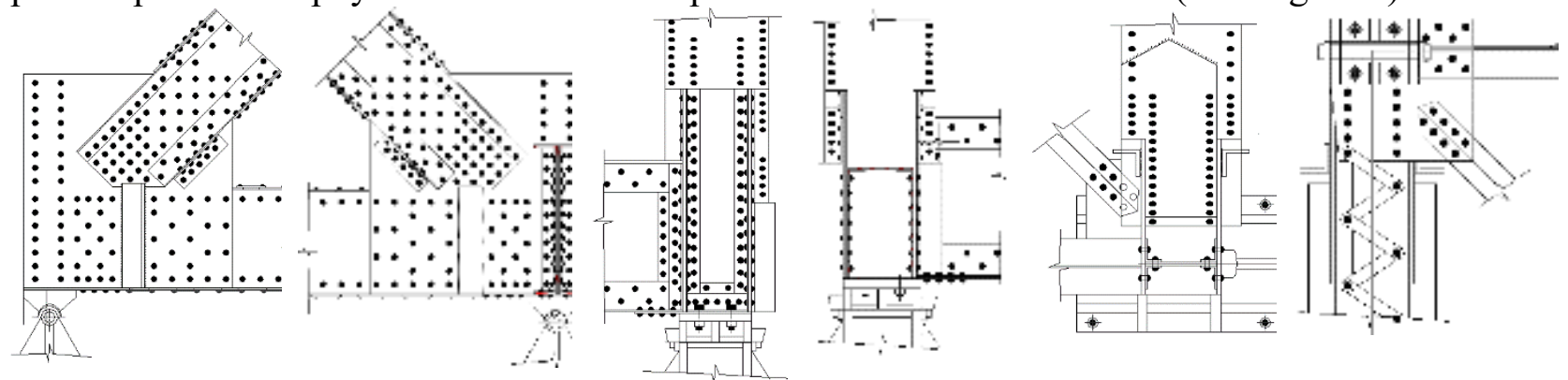

Figura 7: Vistas laterales, posterior, frontal, superior e inferior

Estas uniones coincidentes con las cuatro esquinas del tablero por donde se trasmite la carga a los estribos, asequibles para su revisión y medición; en todos los casos con daños en los elementos inferiores mayoritariamente horizontales aunque también presentan afectados tanto elementos verticales como remaches, angulares y tornillos con alta corrosión y pérdidas de sección además de pérdida del elementos en la unión 1A; la causa fundamental ha sido la falta de limpieza con acumulación de tierra y basura que mantienen la humedad de las aguas de lluvia y ambiental decisiva en el proceso de corrosión.

- A las uniones del cordón superior tramos 1 y 2 - montante - diagonal - portal - cruceta superior (ejes 2 y 10) llegan siete elementos: dos tramos de cordón superior, dos elementos del portal, cruceta superior, montante y diagonal, unidos todos con dos planchas verticales de $20 \mathrm{~mm}$ de espesor y dos planchas horizontales de $10 \mathrm{~mm}$ que mediante remaches consiguen su empotramiento. (Ver Figura 8) 

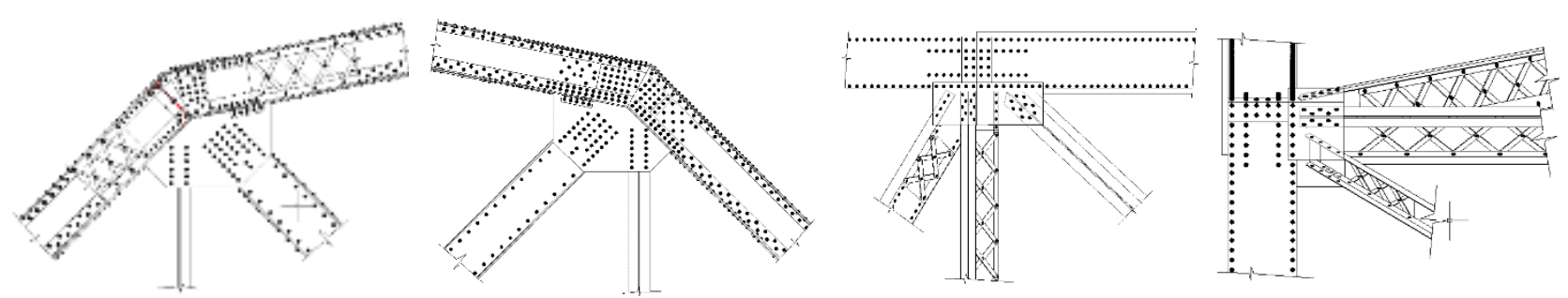

Figura 8: Vistas laterales interior y externa, superior y frontal (Unión 2-A, 2-F, 10-Ay 10-F)
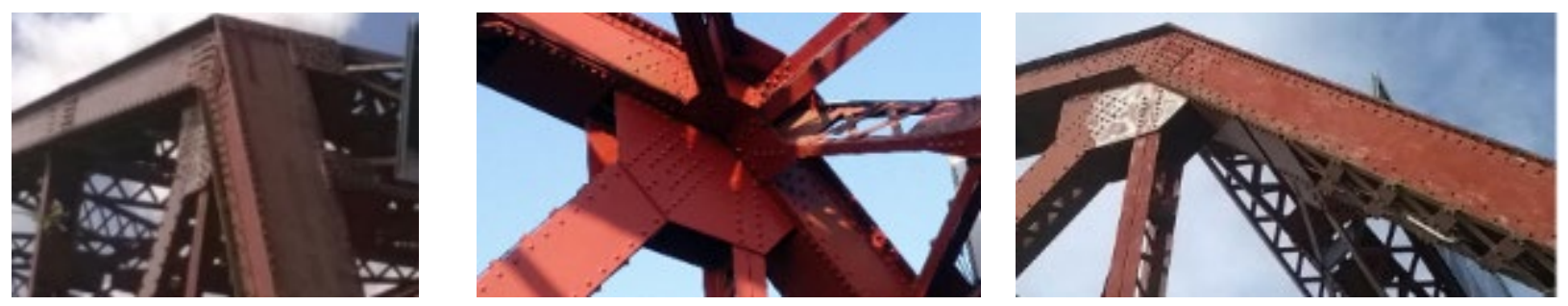

- Unión cordón inferior- montante- viga principal- crucetas inferiores, de cinco elementos, se logra con una lámina inferior fijada al cordón mediante remaches a la que se fijan también con remaches dos crucetas inferiores de arriostre y la viga principal, esta última se une al cordón mediante láminas, cartabones, angulares y remaches, y a su vez con dos láminas paralela exteriores se fija el cordón al montante. (Ver Figura 9)
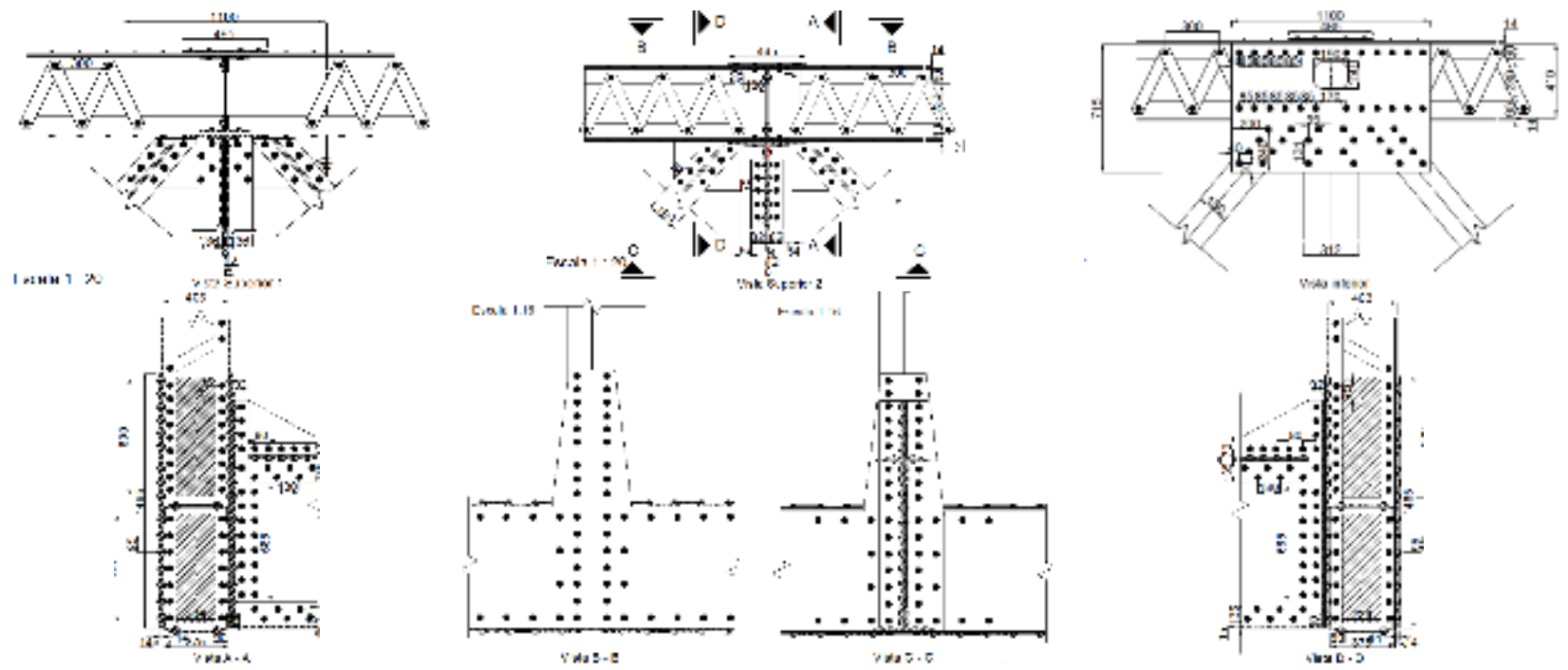

Figura 9 Vistas y secciones de las uniones inferiores 2, 4, 6, 8 y 10 en ambas cerchas.
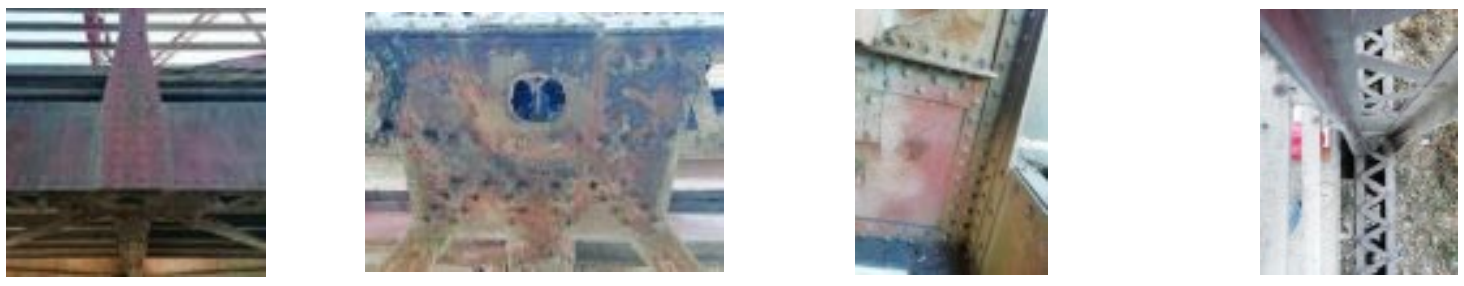

En estos casos de uniones entre elementos de cada cercha y de las cerchas con las vigas principales, como las descritas anteriormente, conseguidas siempre con remaches, y estos, aun 
cuando presentan corrosión con pérdida de sección, no han perdido su función al conservar el fuste excepto algunos casos puntuales, se puede asegurar que todas funcionan garantizando la conexión y empotramiento entre elementos hasta el momento de este estudio. De manera ilustrativa los tres tipos de uniones con las formas de trabajo seguidas, en levantamientos estructurales y patológicos con análisis específicos, explica la forma de trabajo seguida en todo el trabajo.

\subsection{Elementos de cierre}

- Las crucetas superiores, en diagonal, de alrededor de $10.40 \mathrm{~m}$ de longitud y formado por cuatro angulares unidos de manera abierta con riostras que se cruzan conformando una sección "I" de $552 \times 278 \mathrm{~mm}$, están presente en todos los tramos y además de conseguir arriostre y cierre horizontal presenta una sección con peralto tal, que también permiten rigidizar verticalmente a la estructura. Las riostras que en la mayoría de los casos no empatan con el ala arruinando su función rigidizante vertical, en esto pudieron incidir, además de la corrosión localizada en el contacto con los angulares, los impactos por accidentes contra la estructura y las vibraciones que ocurren.
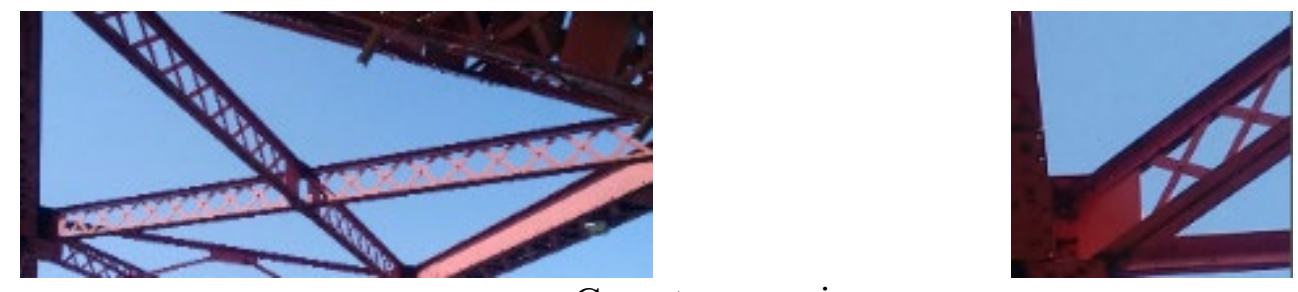

Crucetas superiores

- Las crucetas inferiores, constituidas por dos angulares unidos con remaches que forman una sección " $T$ " invertida, diagonalmente se cruzan en cada tramo rigidizando horizontalmente la estructura, su longitud aproximada es de $10.40 \mathrm{~m}$ y al igual que las crucetas superiores, uno de estos elementos es continuo mientras que el otro son dos tramos que se empalman con una unión empotrada mediante láminas y remaches. Las mayores afectaciones son en sus uniones al cordón inferior con corrosión y pérdida de sección tanto del propio elemento como de la lámina a la que se fija con remaches llegando a pérdida del elemento, con causa fundamental en la falta de limpieza y mantenimiento en su parte más expuesta, no así en el resto del cuerpo del elemento que se conservan muy bien.
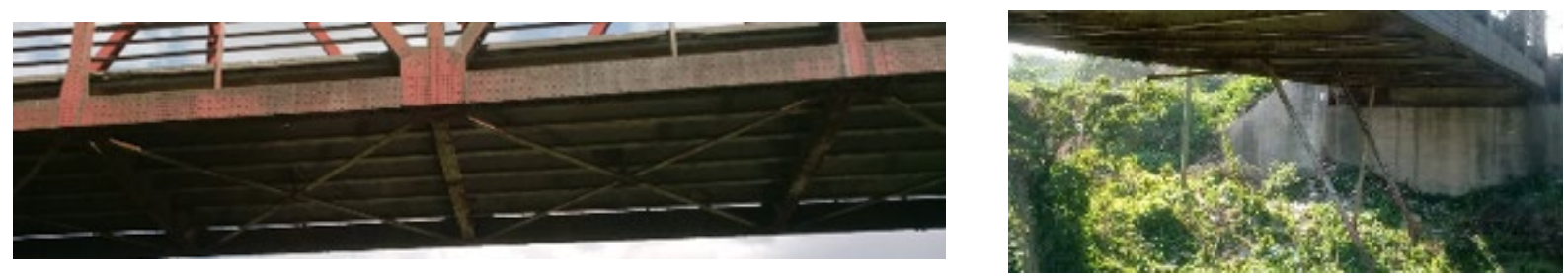

Crucetas inferiores

- Los portales, ubicados en tramos 1 2 y 10 11, en el extremo superior, para unir ambas cerchas, están formados por elementos de sección "I" y sección "C" conformados, al igual que las crucetas superiores, por angulares como alas y alma abierta dada por riostras que se cruzan, que logran la unión entre cerchas mediante dos elementos continuos y dos crucetas interiores empotrados a las cerchas y a los elementos continuos, todos con remaches ocasionando rigidez y monolitismo en la estructura. Ver Figura 10 


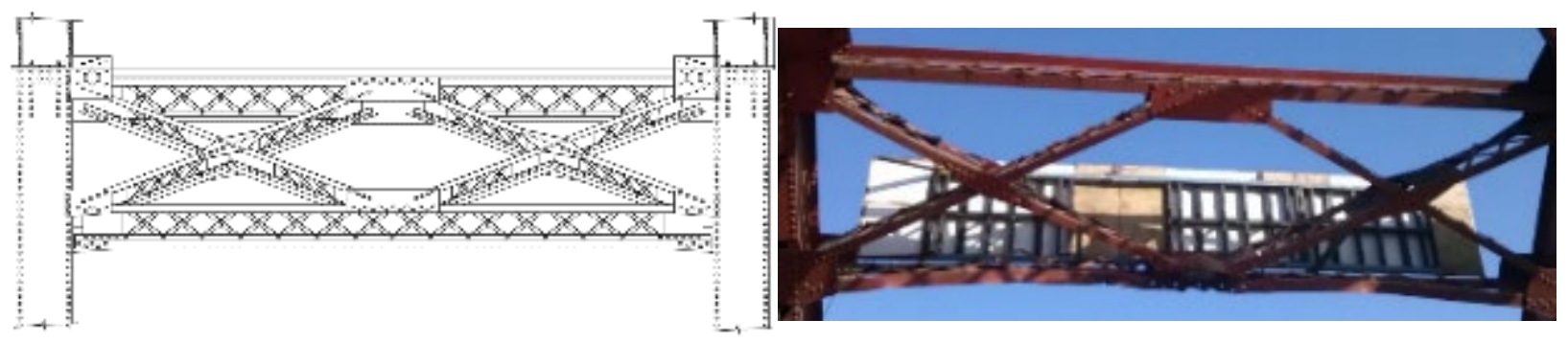

Figura 10 Dibujo y foto del portal a ambos extremos del puente

Estos elementos, aunque mantienen sus funciones de cierre de la estructura, uniendo ambas cerchas para su comportamiento como un todo, han sufrido daños importantes en su cordón inferior y la unión central de este con las crucetas, por golpes de vehículos que superan su altura libre, además, similar a las crucetas, presenta corrosión en los empalmes de las riostras con los cordones que los interrumpe. Estas patologías son comunes en ambos portales

- Contravientos ejes 3 y 9, conformados por angulares que se cruzan, empotran en montantes y cordón superior de ambas cerchas en los ejes 3 y 9, con el objetivo de rigidizar transversalmente la estructura, sus uniones en los cruces también son empotradas con láminas y remaches salvando una altura de $3.70 \mathrm{~m}$ para garantizar el gálibo de $5.00 \mathrm{~m}$. Ver Figura 11
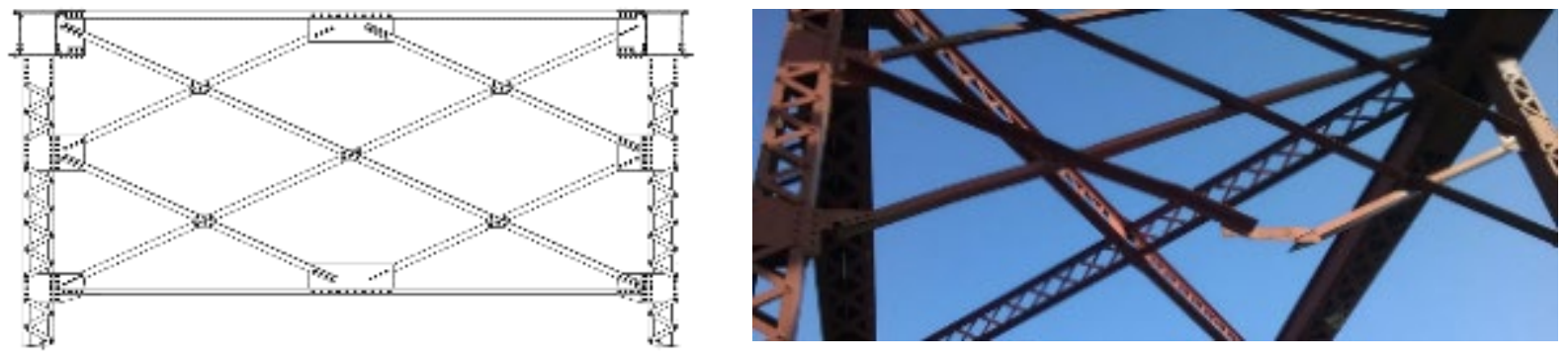

Figura 11 Dibujo y foto de los contravientos ejes 3 y 9

- Contravientos ejes 4 y 8, similar al de los pórticos anteriores son elementos conformados por angulares que se cruzan, empotran en montantes y cordón superior de ambas cerchas en los ejes 4 y 8, para rigidizar transversalmente la estructura, sus uniones en los cruces también son empotradas con láminas y remaches salvando una altura de $5.30 \mathrm{~m}$ para garantizar el gálibo de $5.00 \mathrm{~m}$. Ver Figura 12.
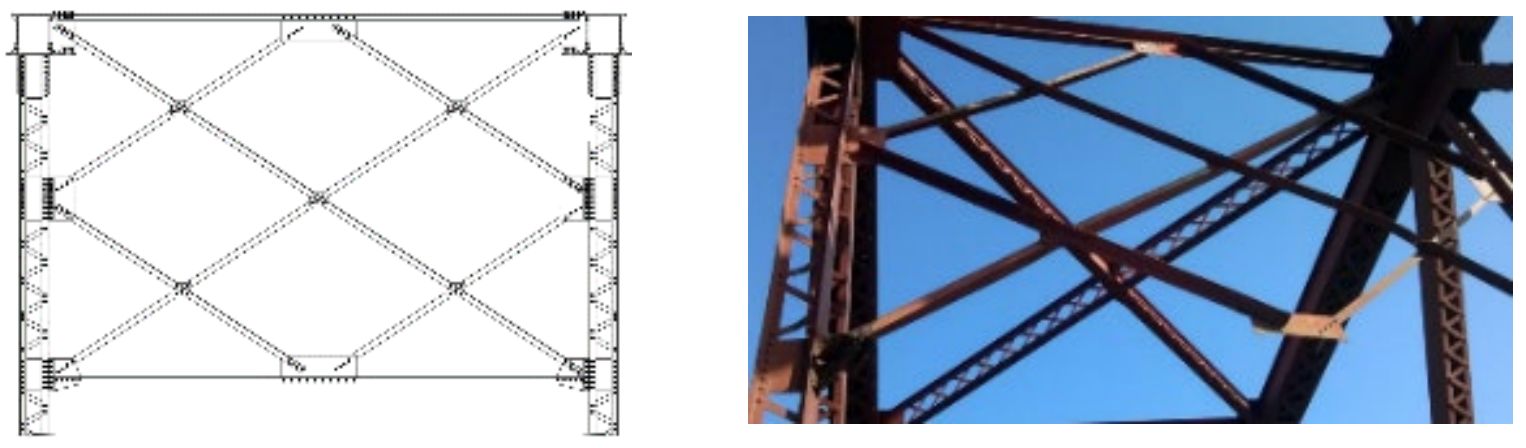
Figura 12 Dibujo y foto de los contravientos ejes 4 y 8

- Contravientos ejes 5 y 7, igual a los anteriores estos contravientos presenta tres uniones en cada lado, dos en montantes y una en cordón superior, donde llegan dos elementos del contraviento en todos los casos, empotradas toda mediante láminas y remaches salvando una altura de $5.85 \mathrm{~m} \mathrm{y}$ gálibo de $5.00 \mathrm{~m}$. Figura 13.
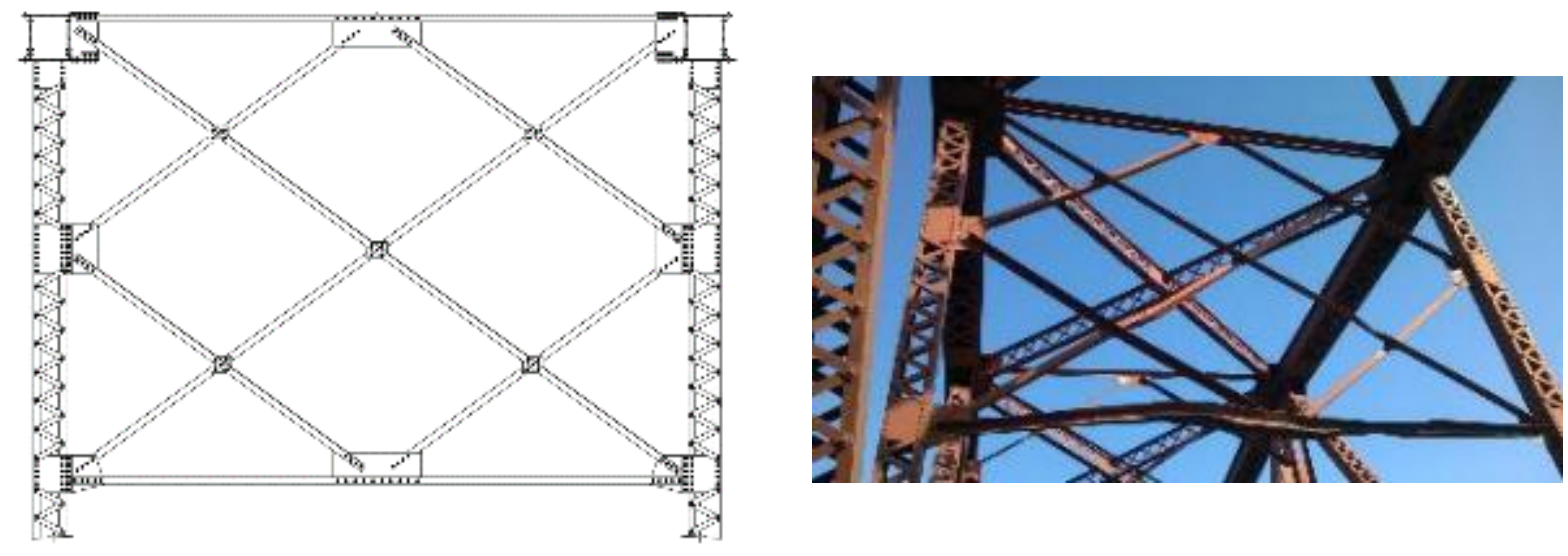

Figura 13 Dibujo y foto de los contravientos ejes 5 y 7

- Contravientos eje 6, elemento vertical de arrostramiento transversal conformado por angulares que se cruzan casi perpendicular, conectando mediante uniones empotradas a ambas cerchas en su tramo más alto con $6.40 \mathrm{~m}$ y altura libre de $5.00 \mathrm{~m}$. (Ver Figura 14)
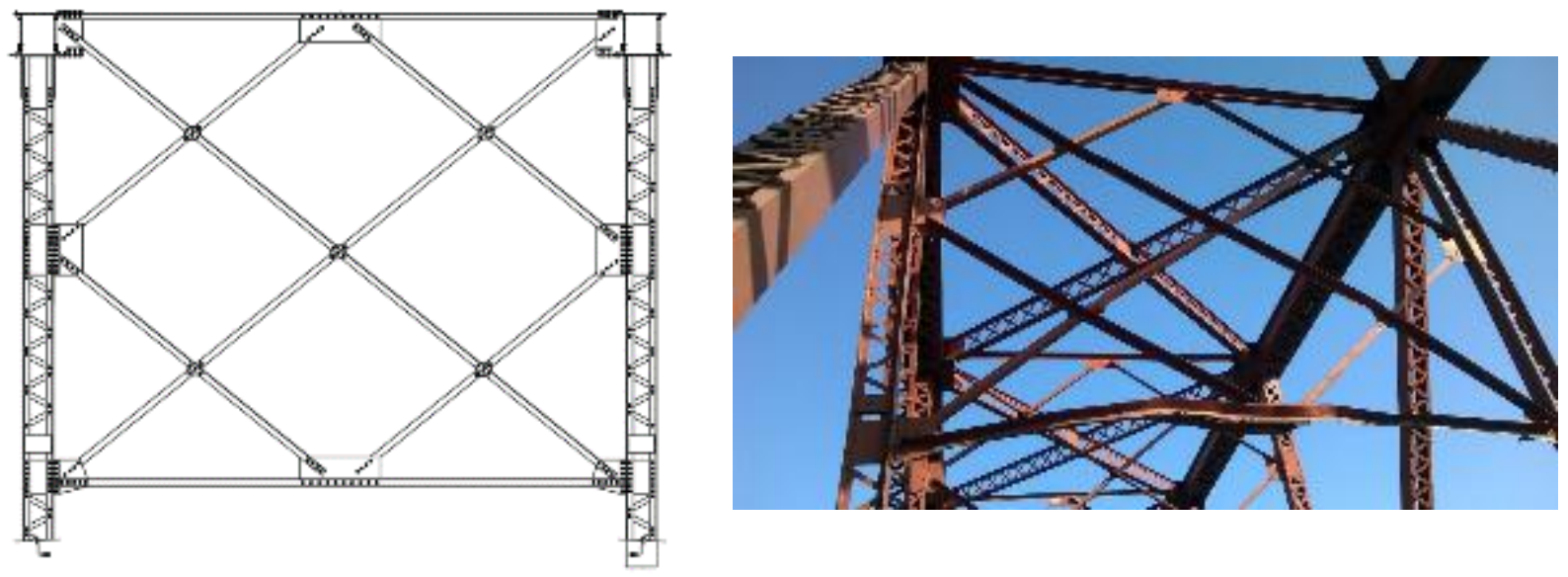

Figura 14: Dibujo y foto del contraviento eje 6

Todos estos elementos de cierre (contravientos), siete en total, presentan daños similares, principalmente los producidos por los golpes, pues al ser estructuras menos reforzadas han sufrido desplazamiento y deformaciones de su entramado llegando a ocasionarles pérdida del cordón inferior y partes de su unión a los montantes sin descartar posible corrosión y corrosión con pérdida de sección en sus uniones 


\section{CONCLUSIONES}

- Las principales causas del deterioro observado en la estructura están en la falta de limpieza y mantenimiento y los impactos ocasionados a la misma por violaciones en la altura de la carga, superiores al gálibo, agravado por la circulación de equipos con carga muy superiores a las de diseño, tiempo de explotación de la obra, irregularidades en el pavimento, junta deficiente entre la obra y el resto de la vía con incidencia en los estribos, ver foto (Grietas de borde Est. Sse) del epígrafe 5.1 y falta de drenaje en la vía entre otros.

- El estado técnico actual de la estructura del puente, critico en los elementos de cierre (crucetas superiores e inferiores, contravientos y portales), requiere de un proyecto de reparación que posibilite restituir la rigidez y comportamiento como un todo de la estructura, minimizando sus vibraciones y oscilaciones; su implementación exigirá de trabajos muy complejos y precisos que requerirán cierres temporales de la obra al tráfico que demanda de una solución vial alternativa.

- Los montantes, como se aprecia en la foto, presentan el efecto de los impactos en los contraviento, deformando sus elementos e incluso desprendiendo su cordón inferior en algunos pórticos con incremento de la altura libre del montante, esto ocasiona además una reducción de la luz libre a la altura del gálibo cuyas mediciones alcanzan valores entre 2 y $9 \mathrm{~cm}$ repartidos aleatoriamente entre $10 \mathrm{dos}$ montantes correspondientes, este fenómeno se observa a simple vista tanto en obra como en fotos aun cuando los valores del ángulo de inflexión creado no supera un grado

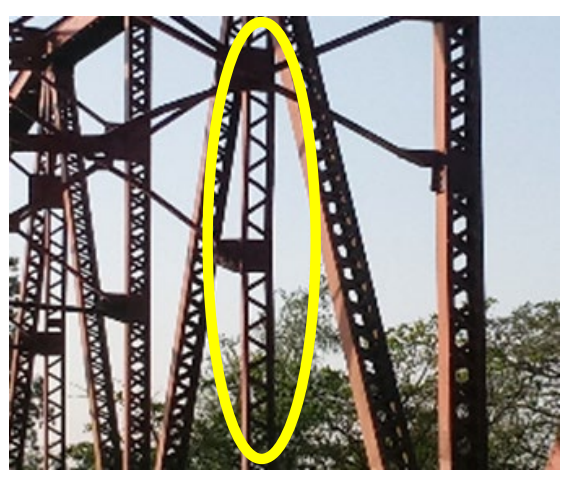
$\left(1^{\circ}\right)$, también debe ocurrir torsión no definidas en este estudio.

- Los aparatos de apoyos han sido otros de los elementos muy afectados por la falta de mantenimiento y limpieza programada, presentando corrosión con pérdida de sección en los cuatro casos, sin embargo, su revisión y observación cuidadosa permiten aseverar que los mismos funcionan, es decir, asimilan las solicitaciones de giro y desplazamiento, que sin mantenimiento pudieran estar ocasionándole desgastes irreversibles. Ver fotos (Pérdidas de sección) del epígrafe 5.2

- El hecho de no disponer de andamios y otros accesorios que facilitaran la inspección y medición detallada de toda la estructura, limitando este trabajo a alrededor de un $45 \%$ del total de los componentes, de existir oscilaciones del valor obtenido en las diferentes medidas producto de las pinturas protectoras, la corrosión, deformaciones, etc, de haberse usados el sistema inglés de medidas en el proyecto original, sin equivalentes exactos en las actuales mediciones en el sistema métrico decimal y atrasos en la información interna entre otros, hacen que la información gráfica obtenida constituida por los diferentes planos confeccionados adquieran en algunos casos carácter de croquis, aunque las medidas acotadas se corresponden con determinaciones reales.

- A pesar de las dificultades afrontadas durante los trabajos de campo detalladas anteriormente, con limitaciones que imposibilitaron alcanzar mayor precisión en la cuantificación de daños a la vez que impiden asignar carácter ejecutivo al informe, mediante vías alternativas, con el uso de binoculares, escalando fotos, vistas escaneadas, etc, aunque no se eliminan, se reducen los niveles de incertidumbre, alcanzando información útil a cualquier etapa de trabajo tanto en el levantamiento estructural como patológico de la obra. 
- En las restricciones de circulación impuestas actualmente por tránsito y vialidad en este tramo, con reducción de la velocidad y circulación en sólo uno de los sentidos del tráfíco, tratando de reducir las vibraciones en la estructura, debe incluirse el trayecto por el centro de la calzada atenuando las solicitaciones transversales a la estructura, pues su pérdida de inercia ha sido en todos los sentidos y afecta el trabajo de conjunto entre las cerchas muy debilitado actualmente.

- Los resultados alcanzado muy útiles aun con las limitaciones de acceso, andamios y equipamiento entren otros aquí destacados, para nada están justificando o aprobando la mala preparación para las investigaciones cuyos resultados exigen niveles de precisión que no den lugar a ambigüedades o confusiones.

- Los trabajos y resultados aquí resumidos con esta combinación de servicios de diferentes disciplinas y distintos especialistas que permitieron alcanzar los objetivos inicialmente trazados constituyen, en este tipo de obra, una estrategia a seguir en estudios venideros de estructuras con similares características.

\section{REFERENCIAS}

Ref. 1. "Manual de Inspección, Evaluación y Diagnóstico de Corrosión en Estructuras de Hormigón

Armado" CYTED 1997

Ref. 2. "Menéndez Menéndez, José. "Desperfectos en obras de ingeniería y arquitectura". Editorial

del Centro de Información de la Construcción”. Ciudad de la Habana, Cuba. 1986.

Ref. 3. Ortega Andrade, Francisco. "Humedades en la edificación". EDITAN, S.A., Sevilla, España.1989.

Ref. 4. Díaz, Marco. "Manual de Estructuras Metálicas". TECNAR

Ref. 5. Serna, Domingo. "El acero en la construcción de estructuras metálicas". Horihuela. Alicante, España.

Ref. 6. "722:2009 Edificaciones y obras civiles- Investigaciones ingenieras aplicadas- Requisitos de alcance y contenido de la documentación técnica".

Ref. 7. "54:269 (1984) Procedimiento para la Ejecución de las Etapas de las Investigaciones Ingeniero Geológicas en Edificaciones". 